\title{
RIMOZIONE DI ENDOTOSSINE BATTERICHE DA CATETERI PER ELETTROFISIOLOGIA CARDIACA CON STERILIZZAZIONE A GAS PLASMA DI $\mathrm{H}_{2} \mathrm{O}_{2}$
}

\author{
Caola I.*, Tessarolo F.***, Caciagli P.*, Guarrera G.**** \\ * Lab. di Microbiologia e Virologia, Osp. S. Chiara, Trento. \\ *** Dip. Ing. Materiali e Tec. Ind., Università di Trento. \\ **** Servizio Cura e Riabilitazione, APSS, Trento
}

Introduzione: I cateteri per elettrofisiologia a ablazione cardiaca che vengono in contatto con il sangue durante l'impiego clinico devono essere apirogeni ( $<20$ UE/dispositivo). Pertanto la quantificazione del carico pirogenico residuo dopo rigenerazione è necessario per garantire un riutilizzo sicuro.

Metodi: A questo scopo si è monitorato lo stato pirogenico di 61 cateteri per cardiologia interventistica nelle tre fasi fondamentali della procedura di rigenerazione: dopo l'utilizzo clinico su paziente, dopo i trattamenti di decontaminazionepulizia e dopo l'intera procedura di rigenerazione che prevede la sterilizzazione mediante gas plasma di perossido di idrogeno.

Risultati: I risultati hanno mostrato che un utilizzo clinico in condizioni igieniche standard non rappresenta una sorgente di contaminazione endotossinica, mentre l'utilizzo di acqua di rubinetto e il lavaggio manuale dei dispositivi può aumentare significativamente il carico pirogenico.

L'applicazione della tecnica di sterilizzazione a gas plasma ha comunque garantito l'abbattimento del carico endotossinico sotto i livelli richiesti dalle normative vigenti in tutti i dispositivi testati, anche in quelli inoculati sperimentalmente in-vitro con 40, 80 o 200 UE per dispositivo.

Conclusioni: Questo risultato suggerisce un ruolo rilevante della tecnica di sterilizzazione adottata nell' abbattimento del carico endotossinico nei dispositivi medici.

Considerando le attuali richieste igieniche nella rigenerazione dei dispositivi medici, la sterilizzazione al gas plasma di perossido di idrogeno andrebbe considerata come un trattamento efficiente nella rigenerazione di cateteri per elettrofisiologia e ablazione cardiaca. 